\title{
High-rate, high-capacity electrochemical energy storage in hydrogen-bonded fused aromatics
}

Tianyang Chen ${ }^{1} \dagger$, Harish Banda ${ }^{1} \uparrow$, Luming Yang ${ }^{1}$, Jian $\mathrm{Li}^{2,3}$, Yugang Zhang ${ }^{4}$, Riccardo Parenti ${ }^{5}$, Mircea Dincă ${ }^{1 *}$

${ }^{1}$ Department of Chemistry, Massachusetts Institute of Technology, Cambridge, MA 02139, United States

${ }^{2}$ Berzelii Center EXSELENT on Porous Materials, Department of Materials and Environmental Chemistry, Stockholm University, 10691 Stockholm, Sweden

${ }^{3}$ Department of Fibre and Polymer Technology, KTH Royal Institute of Technology, Teknikringen 56, 10044 Stockholm, Sweden

${ }^{4}$ Brookhaven National Laboratory, Upton, NY 11973, United States

${ }^{5}$ Automobili Lamborghini S.p.A., 40019 Sant'Agata Bolognese, Italy

*Corresponding author. Email: mdinca@mit.edu

$\dagger$ These authors contributed equally to this work 


\begin{abstract}
:
Designing materials for electrochemical energy storage with short charging times and high charge capacities is a longstanding challenge. The fundamental difficulty lies in installing a high density of redox couples into a stable material that can efficiently conduct both ions and electrons. Here, we report all-organic, fused aromatic materials that store up to $310 \mathrm{mAh} \mathrm{g}^{-1}$ and charge in as little as 33 seconds. This performance stems from abundant quinone/imine functionalities that act as redox-active sites, engage in hydrogen bonding for outstanding stability upon cycling, and enable bulk electronic delocalization for high-rate energy storage. The hydrogen bonding-assisted bulk charge storage here contrasts with the surface-confined or hydration-dependent behavior of traditional inorganic electrodes. These materials outperform state-of-the-art faradaic and capacitive electrodes in both capacity and power capability.
\end{abstract}




\section{Main Text:}

Growing demand for electrifying the transportation sector and decarbonizing the grid requires the development of electrochemical energy storage (EES) systems that cater to a wide variety of energy and power needs $(1,2)$. As the dominant EES devices, lithium-ion cells (LICs) and electrochemical capacitors (ECs) typically only offer either high energy or high power (3). Over the last decade, several attempts were made to achieve high power and energy densities in hybrid energy storage systems (HESSs) by integrating LICs and ECs at the device level (4). This approach represents a modular way of supplying high energy and high power to applications where power demands vary with time or the possibility to recover energy at high power is as important as power delivery itself. However, the complexity and costs involved in device-level engineering diminish the utility of HESSs in compact applications such as electric vehicles. This creates a strong incentive to develop electrode materials that combine the high charge capacity of LICs with the fast charging and long cycling life of ECs.

The combination of high capacity and high power in a single electrode material mandates that the material possesses abundant redox-active sites and that it promotes rapid transport of electrical and ionic charges throughout the electrode bulk. This charge storage mechanism, commonly known as pseudocapacitance, has primarily been identified in redox-active transition metal oxides/nitrides/carbides, which exhibit short charge diffusion lengths when fabricated as thin films (5). However, when film thickness increases with these materials, as required for practicallyrelevant mass loadings, ion and electron transport dramatically decreases, leading to sub-optimal performance (6). Overcoming this challenge typically requires composite synthesis (7) and nanoengineering $(8)$.

Organic materials offer an alternative, more compositionally diverse materials space for designing pseudocapacitive electrodes from earth-abundant elements. However, they typically suffer from poor intrinsic electrical conductivity, a result of limited electronic delocalization, and high solubility in electrolytes, which leads to poor cyclability. These combine to deliver inferior electrochemical performance compared to the inorganic alternatives (9). Although strategies such as polymerization and compositing organic molecules with insoluble admixers can limit electrode dissolution, designing organic materials that are themselves insoluble but still allow swift charge transport and storage is difficult (10). Here, we report bis-tetraaminobenzoquinone (BTABQ) and its polymeric analogue (pBTABQ) as new pseudocapacitive organic materials for EES (Fig. 1A). 
They exhibit a high density of redox-active quinone and imine groups (Fig. 1B) and aromatic backbones with extended conjugation. Owing to strong intermolecular hydrogen bonding and donor-acceptor (D-A) $\pi$ - $\pi$ interactions, they are insoluble in both organic and aqueous media (Fig. 1C). BTABQ and pBTABQ exhibit high charge storage capacities at high charge-discharge rates, which can be associated with rapid pseudocapacitive intercalation processes (Fig. 1D). Charge storage and transport occurs throughout the electrode bulk, even at practical mass loadings, due to extended electronic delocalization and facile ion transport.

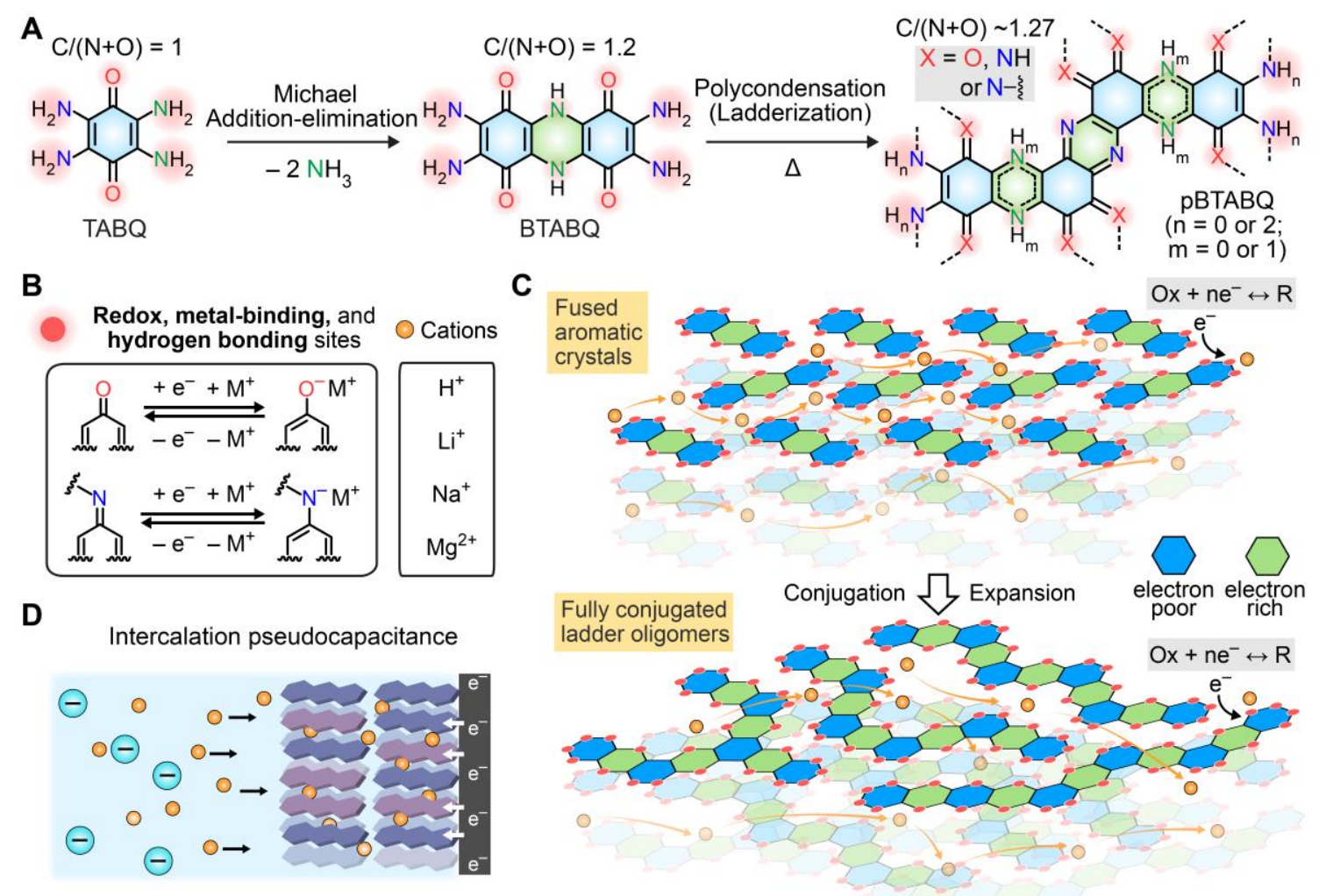

Fig.1. Charge-storage in fused aromatic materials: (A) One-step syntheses of BTABQ and pBTABQ. (B, C) Schematic depiction of fused aromatic molecular crystals and their conversion to conjugated ladder oligomers through self-condensation. The high density of redox-active carbonyl and amine/imine groups enable an alternating arrangement of electron donor (D) and acceptor (A) moieties. The resulting extended conjugation, strong D-A $\pi$ - $\pi$ interactions, and hydrogen bonding facilitate electronic charge delocalization and diffusion of ionic charges. (D) 15 Representation of pseudocapacitive intercalation of various cations into fused aromatic materials. 


\section{Synthesis and characterization}

BTABQ is accessible in gram-quantities by one-step Michael condensation of commercially available tetraaminobenzoquinone (TABQ) (Fig. 1A, S1 and Methods). Although it is highly insoluble in common organic solvents, BTABQ forms as highly crystalline micro-rods (Fig 2A, S2, S3). The crystal structure of BTABQ was obtained from continuous rotation electron diffraction (cRED) using an ab initio method (Fig. 2A) (11), following Pawley refinement of the unit cell parameters from synchrotron powder X-ray diffraction (PXRD) data (fig. S4). Highresolution cRED datasets, down to $0.625 \AA$, allowed direct location of all non-hydrogen atoms (fig. S5, S6, table S1). BTABQ has a planar, fused aromatic backbone. Each BTABQ molecule engages in hydrogen bonding interactions with six neighboring molecules to form twodimensional (2D) layers (Fig. 2B). The 2D layers stack through short D-A $\pi-\pi$ interactions, along the (102) crystallographic direction (fig. S7), to give an interlayer separation of only $3.14 \AA$ (Fig. $2 \mathrm{C}, 2 \mathrm{D})$. The integrity of the $2 \mathrm{D}$ layers is maintained upon exfoliation, as evidenced by scanning electron microscopy (SEM, fig. S8).

Thermogravimetric analysis (TGA) of BTABQ reveals a notable weight loss around $300{ }^{\circ} \mathrm{C}$ (fig. S9). Given the proximity of amino and carbonyl groups in the solid-state structure of BTABQ, we surmised this weight loss may be associated with further condensation between neighboring BTABQ molecules. Indeed, heating bulk BTABQ at $\sim 300{ }^{\circ} \mathrm{C}$ under dynamic vacuum for 2 days yields an amorphous solid with unchanged particle size and morphology (fig. S10-S12). This solid, termed poly(bis-tetraaminobenzoquinone) (pBTABQ), has a significantly higher molecular weight (fig. S13) and lower oxygen and nitrogen content than BTABQ (fig. S14, tables S2, S3), indicating ladderization/polymerization of BTABQ with concomitant loss of water and/or ammonia (12). Pair distribution function (PDF) analyses of both materials reveal similar local structures, confirming that despite the lack of long-range ordering, pBTABQ has a dense solidstate arrangement of molecular fragments analogous to BTABQ (fig. S15). Importantly, Raman spectroscopy revealed broad resonances between $2500 \mathrm{~cm}^{-1}$ and $3100 \mathrm{~cm}^{-1}$ for both BTABQ and pBTABQ, suggesting that the polymer maintains a significant degree of hydrogen bonding (fig. $\mathrm{S} 16)$. $\mathrm{N}_{2}$ sorption studies indicate that both materials are non-porous, with similarly low specific surface areas of $\sim 15-20 \mathrm{~m}^{2} \mathrm{~g}^{-1}$ (fig. S17).

Diffuse-reflectance UV-Vis (DRUV-Vis) spectroscopy revealed that both materials exhibit broad absorption peaks centered around 520 and $800 \mathrm{~nm}$ (Fig. 2E). The former is typical for $\pi-\pi^{*}$ 
intra-quinone electronic transitions (fig. S18A), while extended conjugation within fused aromatic backbones of BTABQ and pBTABQ accounts for the latter (13). Although both materials display significant absorptions in the near-infrared region (NIR), leading to narrow optical band gaps of $0.82 \mathrm{eV}$ and $0.79 \mathrm{eV}$ (Fig. 2E, inset) for BTABQ and pBTABQ, respectively, only the latter has a strong absorption tail into the Mid-IR region. This low energy absorption, rare in organic materials, is indicative of the greater extent of conjugation in $\mathrm{PBTABQ}$ relative to BTABQ, as well as potentially intermolecular electronic excitation processes assisted by strong hydrogen bonding (14) and $\pi-\pi$ stacking (fig. S18B). Altogether, the various analytical data confirm that pBTABQ is a disordered oligomeric analog of BTABQ that has an extended, fused aromatic backbone, maintains a high density of carbonyl/imine redox sites, and supports a persistent hydrogen bonding network in its solid-state packing (see also Supplementary Text).
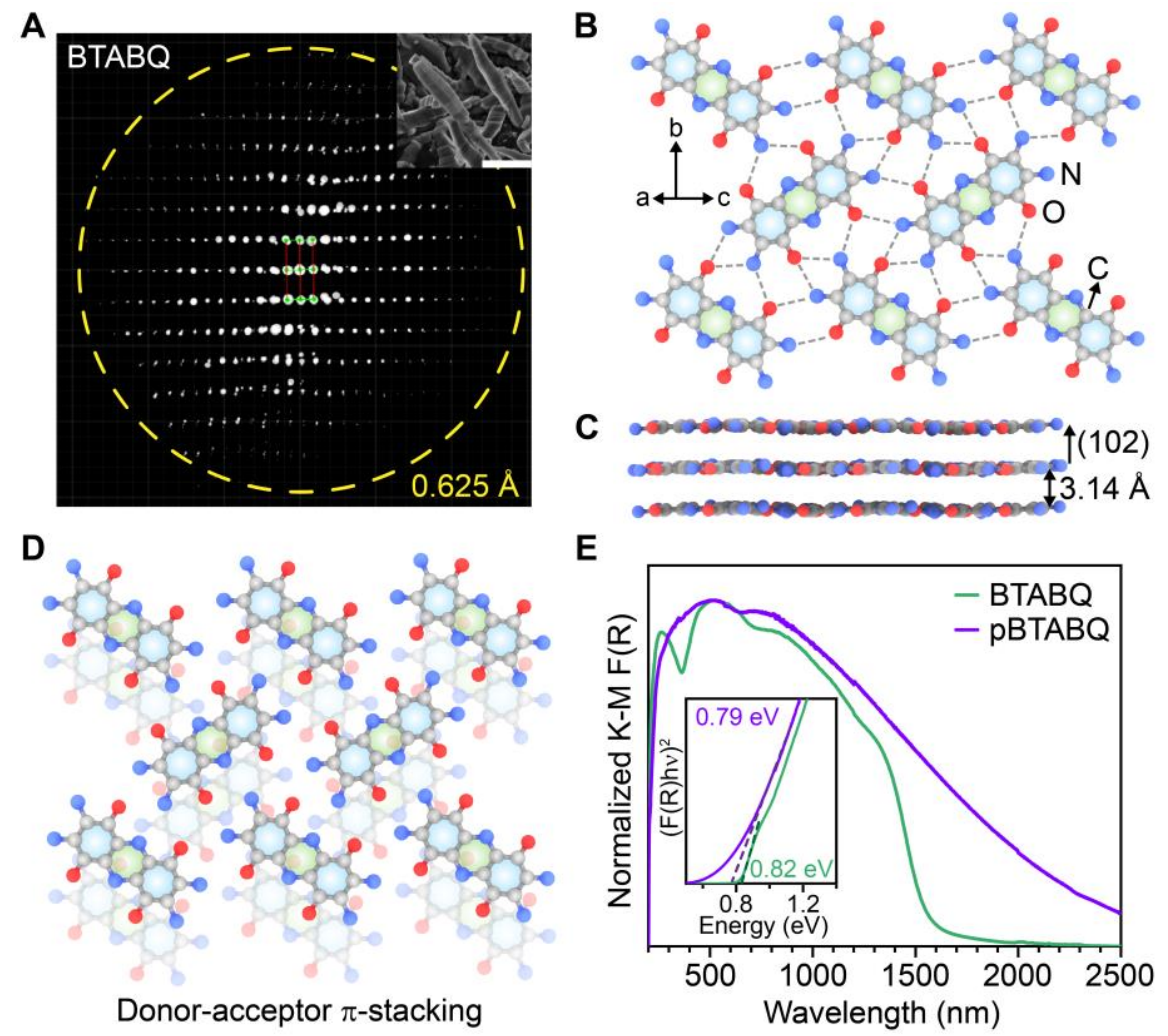

Fig. 2. Characterization of BTABQ and pBTABQ: (A) Projection of 3D reciprocal lattice reconstructed from the cRED dataset of BTABQ with a maximum resolution of $0.625 \AA$. Inset shows a SEM image of BTABQ rods. Scale bar: $2 \mu \mathrm{m}$. (B) A 2D layer of BTABQ molecules formed by hydrogen bonding interactions (dashed lines) between carbonyl and amine/imine groups. The $\mathrm{N} \cdots \mathrm{O}(2.89 \AA)$ and $\mathrm{N} \cdots \mathrm{N}(3.04 \AA)$ distances are shorter than the sum of their van der 
Waals radii $3.15 \AA$ and $3.20 \AA$, respectively. (C) $\pi$ - $\pi$ stacking of 2D layers of BTABQ with an interlayer distance of $3.14 \AA$. (D) Donor-acceptor alignment of 2D layers. (E) DRUV-Vis-NIR spectra of $B T A B Q$ and $\mathrm{pBTABQ}$. Inset shows respective optical band gaps.

BTABQ and pBTABQ exhibit similar bulk electrical conductivities of $2.4 \times 10^{-5} \mathrm{~S} \mathrm{~cm}^{-1}$ and $0.79 \times 10^{-6} \mathrm{~S} \mathrm{~cm}^{-1}$ at room temperature, respectively (fig. S19). Electron paramagnetic resonance (EPR) spectra exhibit intense isotropic signals that are consistent with the presence of organic radicals in both materials (fig. S20), likely stemming from redox-active quinone/imine moieties or the fused heterocyclic systems within BTABQ units. Overall, the presence of a high density of diverse redox sites in extended conjugated materials with efficient electronic delocalization makes $\mathrm{BTABQ}$ and $\mathrm{pBTABQ}$ promising candidates for high-rate EES.

\section{Electrochemical performance in neutral electrolytes}

Cyclic voltammograms (CVs) of BTABQ and $\mathrm{pBTABQ}$, obtained in a three-electrode configuration in a $1 \mathrm{M}$ aqueous solution of $\mathrm{LiCl}$ (see Materials and Methods), display quasirectangular curves at scan rates ranging from 0.2 to $30 \mathrm{mV} \mathrm{s}^{-1}$ (Fig. 3A, S21). Under cathodic polarization, BTABQ and pBTABQ display stable potential windows of $0.5 \mathrm{~V}$ and $1.0 \mathrm{~V}$, respectively. An analysis of the CV currents (i) at different scan rates (v) using the power law equation $\left(i=a v^{b}\right.$ ) reveals $b$ values close to unity for both materials (fig. S22), indicating that the charge storage processes are not limited by ion diffusion (15). Altogether, electrochemical behaviors of $\mathrm{BTABQ}$ and $\mathrm{pBTABQ}$ diverge from the sharp redox features and diffusion-controlled behaviors generally observed for organic molecules with carbonyl or imine groups (10). Instead, their behavior resembles that reported for inorganic materials such as $\mathrm{RuO}_{2} \cdot \mathrm{nH}_{2} \mathrm{O}(16,17)$ and MXenes (18-20). That is, they behave like capacitors but exhibit a rapid redox-based charge storage mechanism known as pseudocapacitance. Indeed, both BTABQ and pBTABQ have low specific surface areas $\left(<20 \mathrm{~m}^{2} \mathrm{~g}^{-1}\right)$, but exhibit high gravimetric specific capacitances of $\sim 510 \mathrm{~F}$ $\mathrm{g}^{-1}$ at $0.2 \mathrm{mV} \mathrm{s}^{-1}$ and $\sim 300 \mathrm{~F} \mathrm{~g}^{-1}$ at $10 \mathrm{mV} \mathrm{s}^{-1}$ (Fig. 3B inset), consistent with a pseudocapacitive charge storage mechanism (21). Galvanostatic charge-discharge (GCD) experiments at current densities ranging from 2 to $10 \mathrm{~A} \mathrm{~g}^{-1}$ display triangular (i.e. capacitive) voltage vs. time profiles (fig. S23). Additionally, both materials display excellent retention of capacitance over 20,000 CV cycles at a scan rate of $30 \mathrm{mV} \mathrm{s}^{-1}$ (Fig. 3B). Furthermore, pBTABQ shows $\sim 96 \%$ retention in GCD experiments at a current density of $10 \mathrm{~A} \mathrm{~g} \mathrm{~g}^{-1}$ for at least 100,000 cycles (fig. S24). 

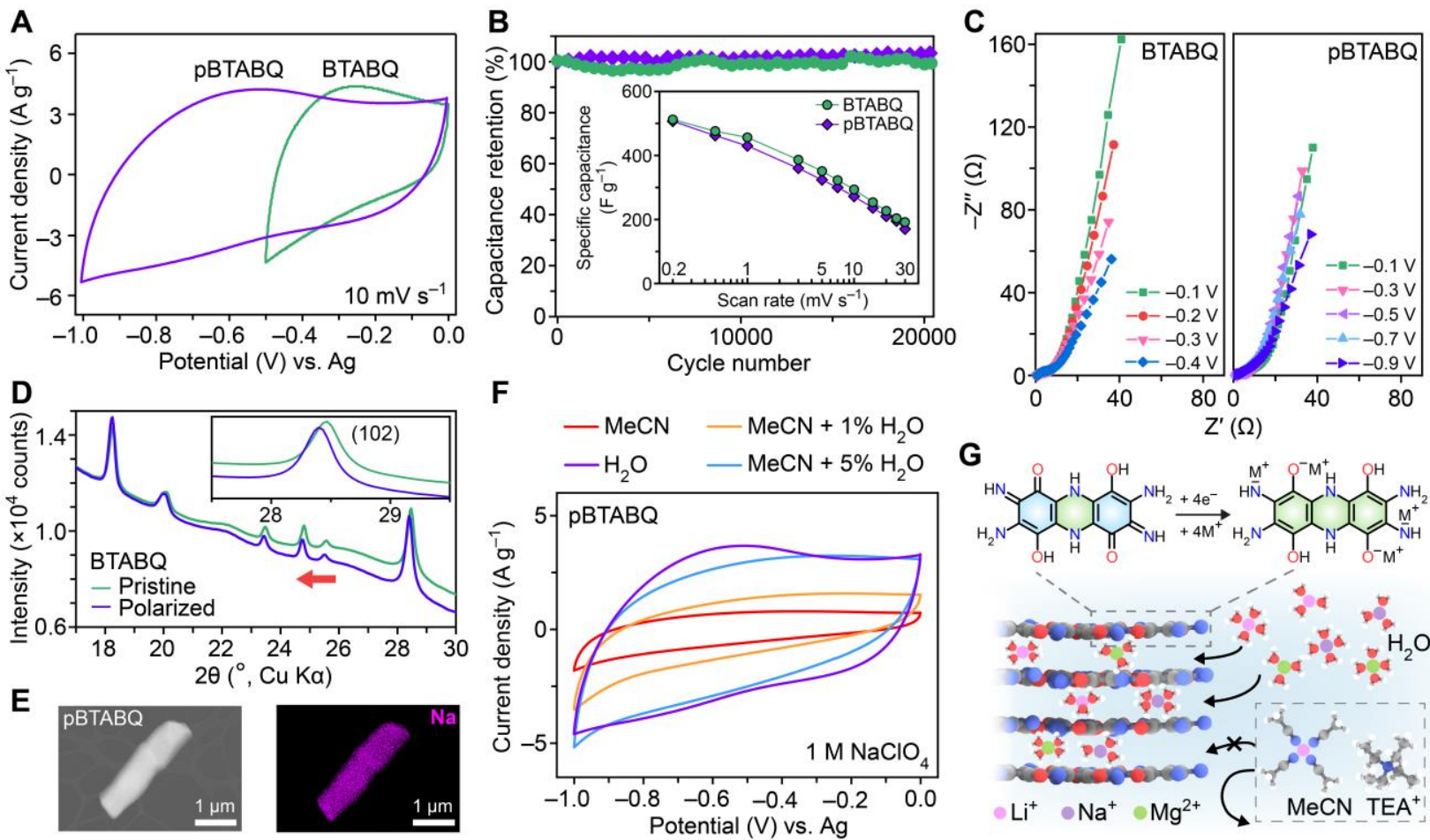

Fig. 3. Charge storage in neutral electrolytes: (A) CVs of BTABQ and pBTABQ obtained at a scan rate of $10 \mathrm{mV} \mathrm{s}^{-1}$ using $1 \mathrm{M} \mathrm{LiCl}$ aqueous electrolyte. (B) BTABQ and pBTABQ retain $~ 98 \%$ of their capacitance over $20000 \mathrm{CV}$ cycles. Inset shows gravimetric specific capacitances at scan rates ranging from 0.2 to $30 \mathrm{mV} \mathrm{s}^{-1}$. (C) EIS of BTABQ and pBTABQ at various applied polarizations reveal typical capacitor-like features with low equivalent series resistances and extended $90^{\circ}$ capacitive regions at low frequencies. (D) In-situ WAXS patterns of pristine and negatively polarized BTABQ reveal lattice expansion, indicating intercalation of ions under polarization. (E) Ex-situ EDS mapping of pBTABQ shows the presence of $\mathrm{Na}^{+}$ions throughout the bulk of a negatively polarized particle. (F) CVs of pBTABQ recorded in electrolytes containing $1 \mathrm{M} \mathrm{NaClO}_{4}$ in mixtures of water and acetonitrile. (G) Schematic representation of water-assisted pseudocapacitive intercalation of cations into BTABQ.

Electrochemical impedance spectroscopy (EIS) provided additional information on the charge storage behavior under dynamic conditions. Nyquist plots of impedance for both BTABQ and pBTABQ (Fig. 3C) display typical capacitor-like features with low equivalent series resistances $(\sim 1-2 \Omega)$, short semicircles and $45^{\circ}$ transition regions along with an extended $90^{\circ}$ capacitive region at low frequencies. Meanwhile, semicircles in the high frequency region reveal decreasing diameters with applied negative potentials (fig. S25), confirming that fast charge transfer events 
accompany charge storage in both materials (22). Overall, EIS and CVs of BTABQ and pBTABQ display capacitor-like features and highlight a possibility of pseudocapacitive charge storage.

Redox processes during charge storage were analyzed through ex-situ X-ray photoelectron spectroscopy (XPS) and solid-state nuclear magnetic resonance (ssNMR) studies of pristine and negatively polarized electrodes (see Methods). The amino functional groups in pristine BTABQ and pBTABQ have partial imine character due to keto-enol tautomerization (Supplementary Text), as verified by the imine carbon chemical shift at 146.4 ppm for BTABQ (fig. S26), and N (1s) XPS spectra of both materials (fig. S27). Deconvolution of O (1s) XPS spectra of polarized electrodes reveal a significant decrease in the intensity of the $\mathrm{C}=\mathrm{O}$ peak and the appearance of a $\mathrm{C}-\mathrm{O}^{-}$peak, indicating the reduction of carbonyl groups (fig. S27). Deconvolution of N (1s) spectra indicates the disappearance of imine component and the growth of a benzoid-amine component upon reduction. The ssNMR spectrum of polarized BTABQ (fig. S26) further supports that both carbonyl and imine groups are affected by reduction and are thus likely responsible for charge storage in BTABQ and pBTABQ (Fig. 3G and Supplementary Text). Charges stored at these redox sites are delocalized both intramolecularly within the fused aromatic backbones, and intermolecularly through hydrogen bonding and D-A $\pi$ - $\pi$ stacking, manifesting in distinctly quasirectangular CVs. In contrast, small organic molecules with localized electronic structures display well-defined and sharp redox features $(9,10)$. Similarly, larger $\pi$-conjugated organic molecules with close $\pi$ - $\pi$ stacking, such as 5,7,12,14-pentancetetrone $(23,24)$, that lack hydrogen bonding interactions also exhibit limited electronic delocalization (fig. S28), leading to sharp, wellseparated CV peaks (fig. S29).

Wide-angle X-ray scattering (WAXS) and energy-dispersive X-ray spectroscopy (EDS) provided insight into whether the pseudocapacitance in BTABQ and pBTABQ is best described as surface-confined or as an intercalation-based bulk process (5). WAXS patterns for BTABQ 25 samples negatively polarized in-situ display a notable shift of the (102) reflection toward a lower $2 \theta$ value (Fig. 2C, 3D, S30, S31). This corresponds to an increase in spacing between 2D layers of BTABQ during $\mathrm{Li}^{+}$insertion, and supports an intercalation-based charge storage mechanism. Remarkably, other cations such as $\mathrm{Na}^{+}$and $\mathrm{Mg}^{2+}$ can also intercalate into both materials, as verified by ex-situ EDS studies of negatively polarized samples (Fig. 3E, S32, S33). Elemental mapping indicates that a significant amount of metal cations uniformly distributes throughout the particles, confirming charge storage in the bulk of both BTABQ and pBTABQ electrodes. 
Studies in a series of different electrolyte solutions provided important information on the role of electrolytic ions and solvents on intercalation. Various alkali or alkaline earth metal ions did not cause significant changes in the CVs for either material (fig. S34). However, markedly lower currents were observed with electrolytes containing tetraethylammonium (TEA ${ }^{+}$) (fig. S35). Partially substituting $\mathrm{TEA}^{+}$with increasing amounts of $\mathrm{Li}^{+}$led to corresponding increases in current. Altogether, these results show that both materials can intercalate a variety alkali and alkaline earth ions but exclude larger TEA ${ }^{+}$ions, presumably based on size (Fig. 3G). The ability of BTABQ and pBTABQ to intercalate multiple cations and display size-differentiated pseudocapacitive behavior mimics that of MXenes $(18,20)$ and coordination polymers $(24)$.

Solvent also plays a critical role in intercalation. CVs of pBTABQ recorded in a $1 \mathrm{M}$ solution of $\mathrm{NaClO}_{4}$ in acetonitrile (MeCN) exhibit substantially lower currents than those recorded in an aqueous solution with identical concentration of $\mathrm{NaClO}_{4}$ (Fig. 3F). Addition of $5 \%$ water to $\mathrm{MeCN}$ led to essentially quantitative recovery of capacitive current with $\mathrm{NaClO}_{4}$, but did not have an effect for electrolytes made with $\mathrm{TEA}^{+}$(fig. S36). These results suggest a critical role for water during cation intercalation into both $\mathrm{BTABQ}$ and $\mathrm{pBTABQ}$. This behavior is similar to that observed in vanadium oxides (26-28) and Prussian blue analogues (PBAs) (29) and has been rationalized as water assisting intercalation by lowering both the energy barrier for solid-state ion diffusion and the desolvation penalty at the electrode-electrolyte interface (Fig. 3G).

\section{Influence of $\mathrm{pH}$ on charge storage}

The $\mathrm{pH}$ of electrolytes often strongly modulates the charge storage behavior of pseudocapacitive materials $(17,30)$. With evidence in hand that both hydrogen bonding and water critically influence the electrochemical response of both BTABQ and pBTABQ, we evaluated the influence of $\mathrm{pH}$ using aqueous $\mathrm{NaCl}$ electrolytes in the $\mathrm{pH}$ range $0-14.7$ (see Methods). Although both materials exhibit similar dependence on $\mathrm{pH}$, the discussion below is primarily focused on pBTABQ, given its larger electrochemical stability window $(1.0 \mathrm{~V})$. 

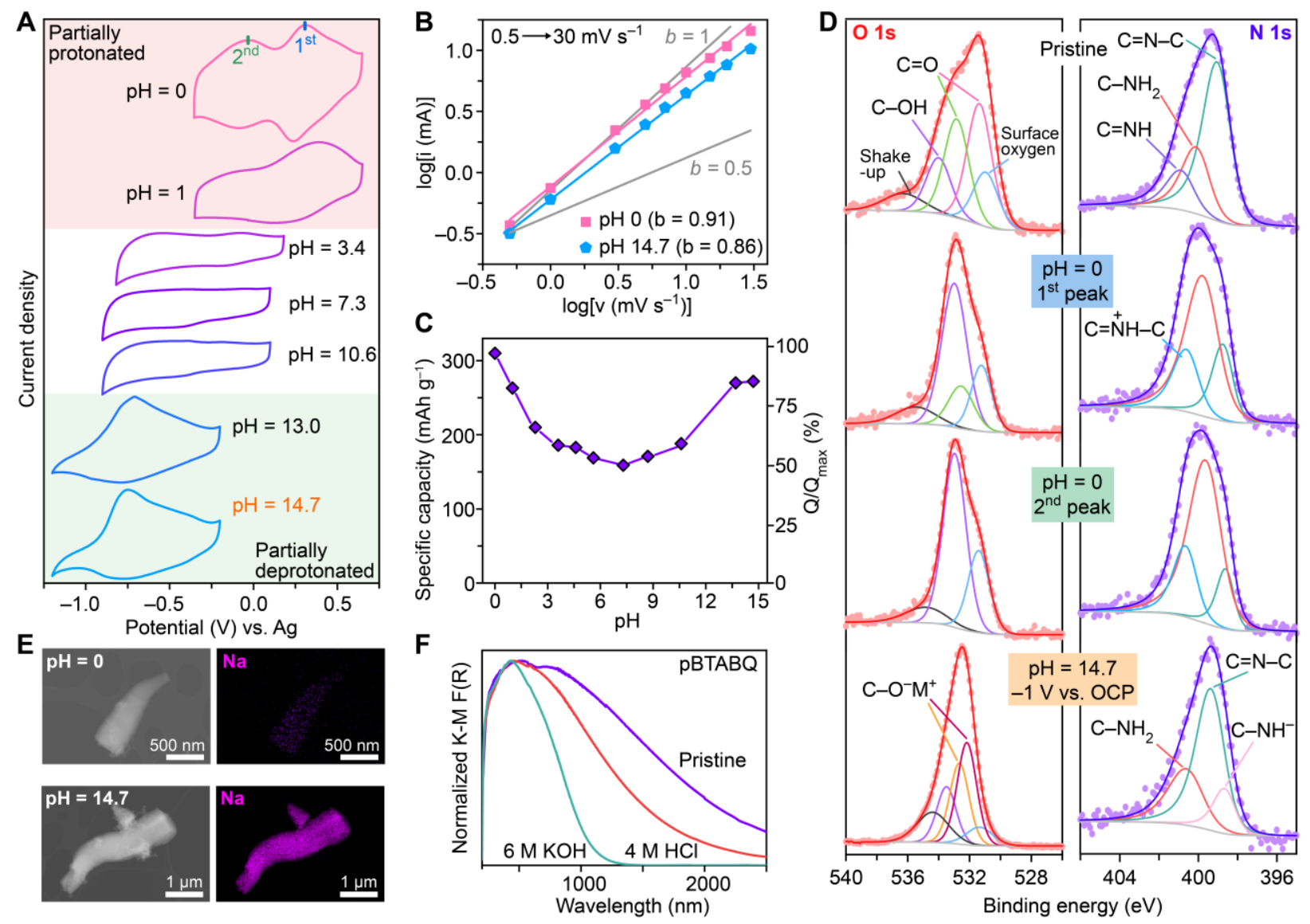

Fig. 4. Role of pH on charge storage in pBTABQ: (A) CVs obtained at a scan rate of $5 \mathrm{mV} \mathrm{s}^{-1}$ in aqueous $1 \mathrm{M} \mathrm{NaCl}$ electrolyte solutions buffered at $\mathrm{pH}$ values ranging from 0 to 14.7. (B) Peak current versus scan rate analysis of the observed peaks under highly acidic and alkaline conditions reveal $b$ values close to unity. (C) Plot of gravimetric specific capacities as a function of electrolyte $\mathrm{pH}$ obtained from $\mathrm{CVs}$ at a scan rate of $0.2 \mathrm{mV} \mathrm{s}^{-1}$. (D) Ex-situ high resolution $\mathrm{O}$ (1s) and $\mathrm{N}$ (1s) XPS of polarized electrodes highlight changes at $\mathrm{C}=\mathrm{O}$ and $\mathrm{C}=\mathrm{N}$ groups at $\mathrm{pH}=0$ and 14.7. (E) EDS mappings of polarized $\mathrm{pBTABQ}$ under at $\mathrm{pH}=0$ and 14.7. (F) DRUV-Vis spectra of pristine and $\mathrm{pBTABQ}$ and after soaking in solutions of $4 \mathrm{M} \mathrm{HCl}$ or $6 \mathrm{M} \mathrm{KOH}$.

CVs recorded in electrolytes with intermediate $\mathrm{pH}(3.0$ - 12.0) remain quasi-rectangular, whereas CVs recorded under highly acidic or alkaline conditions exhibit broad redox features with small peak separations and markedly increased current (Fig. 4A). Specifically, two sets of broad redox peaks are noted at $\mathrm{pH}=0-1$, whereas a single broad redox feature is observed at $\mathrm{pH}>13$ (fig. S37). Analysis of peak currents vs. scan rates (vide supra) finds $b$ values of $\sim 0.9$ at $\mathrm{pH}<1$ and $\mathrm{pH}>13$ (Fig. 4B), indicating that even at these $\mathrm{pH}$ extremes, charge storage is not limited by 
bulk ion diffusion. The total amount of charge stored at different $\mathrm{pH}$, calculated as gravimetric specific capacity $\left(\mathrm{mAh} \mathrm{g}^{-1}\right.$ ) from CVs at $0.2 \mathrm{mV} \mathrm{s}^{-1}$ (see Methods), increases from $\sim 160 \mathrm{mAh} \mathrm{g}^{-1}$ at intermediate $\mathrm{pHs}$ to 272 and $310 \mathrm{mAh} \mathrm{g}^{-1}$ at $\mathrm{pH}$ of 14.7 and 0 , respectively (Fig. 4C, S38). This "U-shaped" $\mathrm{pH}$-dependence of specific capacities has previously been observed for $\mathrm{RuO}_{2} \cdot x \mathrm{H}_{2} \mathrm{O}$ (17), but, to our knowledge, is a first in organic systems. Remarkably, the observed capacities are substantially higher than state-of-the-art inorganic pseudocapacitive electrodes and higher even than LIC cathodes that function at comparable electrochemical potentials $(5,31)$. Moreover, high capacities of $225 \mathrm{mAh} \mathrm{g}^{-1}(\mathrm{pH}=0), 135 \mathrm{mAh} \mathrm{g}^{-1}(\mathrm{pH}=14.7)$, and $72 \mathrm{mAh} \mathrm{g}^{-1}(\mathrm{pH}=7.3)$ can be accessed at fast rates, within 33 seconds (fig. S39). Polymeric pBTABQ exhibits best rate capability at $\mathrm{pH}=0$, with retention of $96 \%, 90 \%$ and $70 \%$ capacities relative to the highest experimental capacity of $320 \mathrm{mAh} \mathrm{g}^{-1}\left(\mathrm{Q}_{\max }, 0.05 \mathrm{mV} \mathrm{s}^{-1}\right.$, fig. S40), at increasing scan rates of $0.2,5$ and $100 \mathrm{mV} \mathrm{s}^{-1}$, respectively (fig. S39). Assuming a minimum of three BTABQ units in every oligomeric pBTABQ unit, one can estimate a 12-electron reduction and a theoretical maximum capacity of $389 \mathrm{mAh} \mathrm{g}^{-1}$ (see Fig. 3G and Supplementary Text) for pBTABQ, such that 15 the experimental bulk utility of $\mathrm{pBTABQ}$ at $\mathrm{pH}=0$ is $82 \%$. Remarkably, GCD tests at $\mathrm{pH}=0$ under high current densities of 2-10 $\mathrm{A} \mathrm{g}^{-1}$ (fig. S41) also deliver capacities greater than $200 \mathrm{mAh}$ $\mathrm{g}^{-1}$ at rates corresponding to 6-50 $\mathrm{C}(1 \mathrm{C}=$ discharge in 1 hour $)$.

Ex-situ studies on polarized electrodes provided information on the nature of the redox peaks leading to high capacities under acidic and alkaline conditions. First, a decrease in the intensity of the $\mathrm{C}=\mathrm{O}$ and $\mathrm{C}=\mathrm{N}$ components of the $\mathrm{O}(1 \mathrm{~s})$ and $\mathrm{N}(1 \mathrm{~s})$ XPS signals, respectively, upon polarization (Fig. 4D) suggests that charge storage still occurs through the reduction of imines and carbonyls. A notable difference under highly acidic conditions is the absence of alkaline ions by elemental mapping (Fig. 4E) and by XPS (fig. S42), which suggests that protons are exclusively responsible for charge storage under these conditions. Second, the emergence of distinguishable 25 redox peaks suggests the presence of localized redox states, likely arising from weakened electronic delocalization through partial protonation or deprotonation of pBTABQ under highly acidic or alkaline conditions. Indeed, the UV-Vis spectra of pBTABQ soaked in strong acid or base show significantly blue-shifted absorption (Fig. 4F), as expected for diminished charge delocalization. XPS analysis (Fig. 4D, S43) indicates that under acidic conditions, redox processes are proton-assisted, with the first peak involving both imines and carbonyls, and the second peak involving only carbonyl moieties. The broad peak observed under alkaline conditions can instead be assigned to the formation of both enolates and benzoid-amine (Fig. 4D). The exclusive 
involvement of protons at the expense of metal cations at high acidity implies excellent bulk proton diffusion under these conditions, likely promoted by the hydrogen bonding network of pBTABQ (fig. S44). However, the same argument cannot explain the considerable increase in specific capacity at $\mathrm{pH}>13$, where the concentration of protons is very low. Under these conditions, intercalation of $\mathrm{Na}^{+}$dominates, with a substantially larger amount of $\mathrm{Na}$ evident by EDS under alkaline conditions than under neutral conditions (Fig. 4E, S45, S46, Table S4). The seemingly anomalous increase in capacities at the $\mathrm{pH}$ extremes highlights the importance of combining intercalation with a flexible hydrogen-bonded structure that either promotes proton transport at low $\mathrm{pH}$, or allows deprotonation at high $\mathrm{pH}$, thus allowing metal cation intercalation (29).
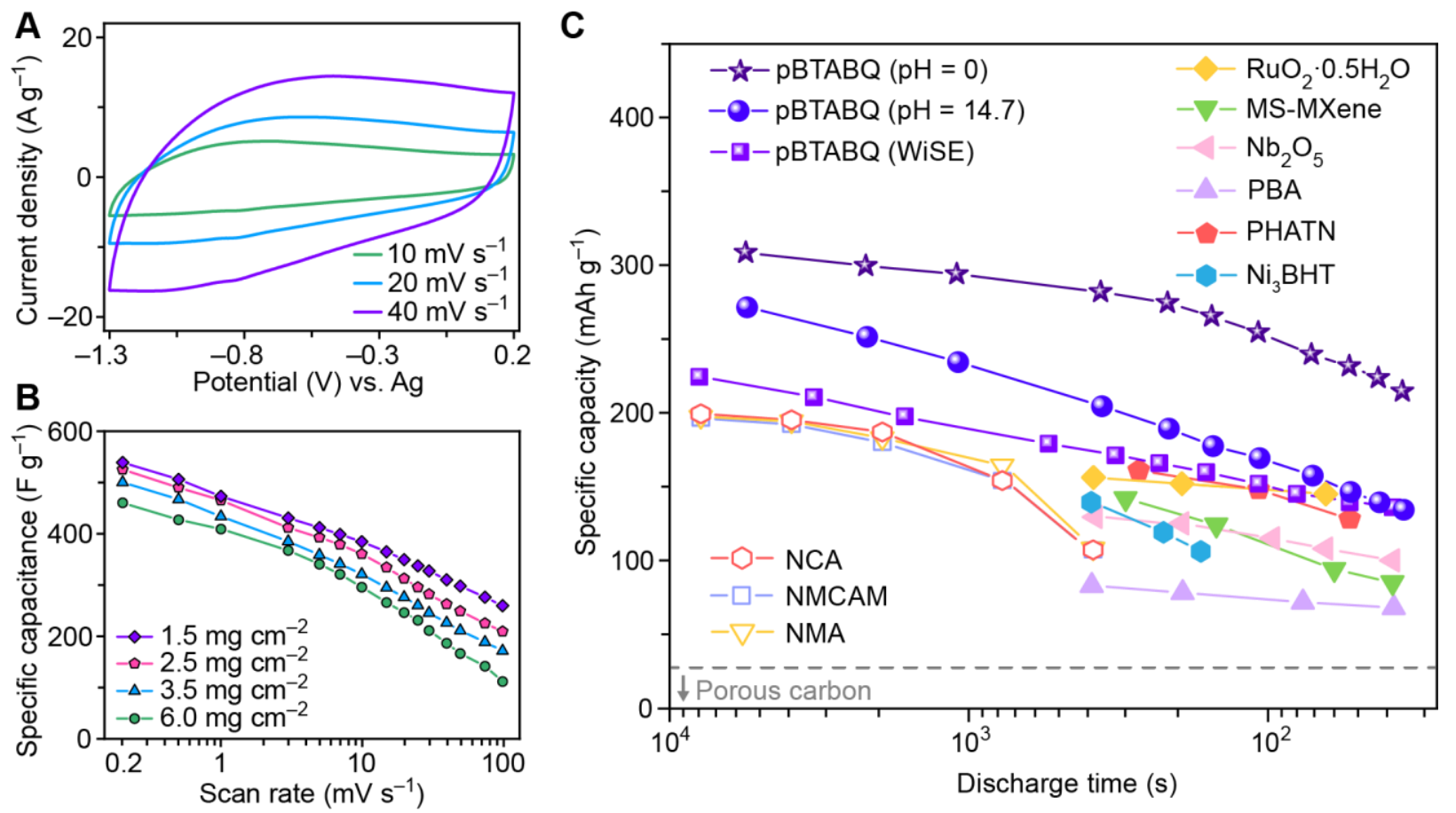

Fig.5. Electrochemical studies in water-in-salt electrolytes (WiSE) and performance benchmarking. (A) CVs of pBTABQ obtained at scan rates of 10, 20, and $40 \mathrm{mV} \mathrm{s}^{-1}$ using 17 molal $\mathrm{NaClO}_{4} \mathrm{WiSE}$ electrolyte. (B) Power performance of pBTABQ in WiSE obtained from CVs at scan rates ranging from 0.2 to $100 \mathrm{mV} \mathrm{s}^{-1}$ on electrodes with active material loadings of 1.5, 2.5, 3.5 , and $6 \mathrm{mg} \mathrm{cm}^{-2}$. (C) Comparison of rate performances for pBTABQ and related state-of-theart LIC cathodes $(29,31)$ or pseudocapacitive $(15,19,21,32,33)$ electrodes. Materials tested at potentials greater than $2 \mathrm{~V}$ vs. $\mathrm{Li}^{+} / \mathrm{Li}$ and with a minimum active material loading of $1.5 \mathrm{mg} \mathrm{cm}^{-2}$ are chosen for comparison. MS-Mxene: $\mathrm{Ti}_{3} \mathrm{C}_{2} \mathrm{~T}_{x}$ synthesized using Molten alkali salts, PHATN: 
perylene diimide-hexaazatrinaphthylene, NCA, NMCAM and NMA: Ni-rich analogues of $\mathrm{LiNiO}_{2}$ doped with $\mathrm{Al}, \mathrm{Mn}, \mathrm{Co}$ and $\mathrm{Mg}, \mathrm{Ni}_{3} \mathrm{BHT}$ : $\mathrm{Ni}_{3}$ (benzenehexathiol).

\section{Deep charge-discharge capability}

Benefiting from the recent development of water-in-salt electrolytes (WiSE) $(34,35)$, we

explored deep charge-discharge capability of pBTABQ in a larger reductive potential window of $1.5 \mathrm{~V}$. CVs recorded in 17 molal $\mathrm{NaClO}_{4}$ remain quasi-rectangular and deliver capacitances similar to those seen in aqueous neutral electrolytes, with over $90 \%$ capacity retention over 40,000 cycles (Fig. 5A, fig. S47). The corresponding Nyquist plots recorded under various negative polarizations confirm the capacitor-like features, as with those recorded in aqueous neutral electrolytes (fig. S48). Notably, EDS maps of polarized pBTABQ electrodes in WiSE reveal a significantly larger amount of intercalated $\mathrm{Na}^{+}$ions relative to that observed in neutral electrolyte (Fig. S49, Table $\mathrm{S} 4)$. This indeed is reflected in a higher charge capacity of $225 \mathrm{mAh} \mathrm{g}^{-1}$ for the WiSE system, a $50 \%$ increase relative to neutral aqueous electrolytes. Rate capability studies display ultrahigh-rate performance at various active material loadings as high as $6 \mathrm{mg} \mathrm{cm}^{-2}$, a practical loading value (Fig. 5B). For instance, discharge capacities greater than $100 \mathrm{mAh} \mathrm{g}^{-1}$ can be delivered within 60 seconds. A comparison between the performance of pBTABQ in acidic, alkaline, or WiSE electrolytes and state-of-the-art high-capacity LIC cathodes and high-rate pseudocapacitive electrodes is shown in Fig. 5C. In a full cell, an asymmetric hybrid capacitor fabricated using pBTABQ as the negative electrode and porous activated carbon as the positive electrode delivers $2 \mathrm{~V}$ and cycles safely over 60,000 cycles with a capacitance retention of over 90\% (fig. S50, 51). Optimization of the WiSE electrolyte (see Methods) (36) further enhances the cell voltage to 2.3 V (fig. S52) and highlights additional opportunities for device-level improvements. Overall, the new materials reported here and the structural and compositional principles underlining their electrochemical performance provide important blueprints toward the use of organic

25 pseudocapacitive materials in practical, high-rate, high-capacity EES devices.

\section{References and Notes}

1. P. Simon, Y. Gogotsi, Perspectives for electrochemical capacitors and related devices. Nat. Mater. 19, 1151-1163 (2020).

2. T. M. Gür, Review of electrical energy storage technologies, materials and systems: challenges and prospects for large-scale grid storage. Energy Environ. Sci. 11, 2696-2767 
(2018).

3. P. Simon, Y. Gogotsi, B. Dunn, Where do batteries end and supercapacitors begin? Science 343, 1210-1212 (2014).

4. Z. Zou, J. Xu, F. Zeng, X. Mei, A hybrid energy storage system for dual-motor driven electric vehicles. DEStech Trans. Environ. Energy Earth Sci. (iceee). 145-148 (2019).

5. C. Choi, D. S. Ashby, D. M. Butts, R. H. DeBlock, Q. Wei, J. Lau, B. Dunn, Achieving high energy density and high power density with pseudocapacitive materials. Nat. Rev. Mater. 5, 5-19 (2020).

6. S. Fleischmann, J. B. Mitchell, R. Wang, C. Zhan, D. Jiang, V. Presser, V. Augustyn, Pseudocapacitance: from fundamental understanding to high power energy storage materials. Chem. Rev. 120, 6738-6782 (2020).

7. H. Sun, L. Mei, J. Liang, Z. Zhao, C. Lee, H. Fei, M. Ding, J. Lau, M. Li, C. Wang, X. Xu, G. Hao, B. Papandrea, I. Shakir, B. Dunn, Y. Huang, X. Duan, Three-dimensional holey-graphene/niobia composite architectures for ultrahigh-rate energy storage. Science 356, 599-604 (2017).

8. Y. Xia, T. S. Mathis, M. Zhao, B. Anasori, A. Dang, Z. Zhou, H. Cho, Y. Gogotsi, S. Yang, Thickness-independent capacitance of vertically aligned liquid-crystalline MXenes. Nature 557, 409-412 (2018).

9. P. Poizot, J. Gaubicher, S. Renault, L. Dubois, Y. Liang, Y. Yao, Opportunities and challenges for organic electrodes in electrochemical energy storage. Chem. Rev. 120, 6490-6557 (2020).

10. Y. Lu, Q. Zhang, L. Li, Z. Niu, J. Chen, Design strategies toward enhancing the performance of organic electrode materials in metal-ion batteries. Chem 4, 2786-2813 (2018).

11. Z. Huang, E. S. Grape, J. Li, A. K. Inge, X. Zou, 3D electron diffraction as an important technique for structure elucidation of metal-organic frameworks and covalent organic frameworks. Coord. Chem. Rev. 427, 213583 (2021).

12. L. Jongbok, A. J. Kalin, T. Yuan, M. Al-Hashimi, L. Fang, Fully conjugated ladder polymers. Chem. Sci. 8, 2503-2521 (2017). 
13. H. Audi, Z. Chen, A. Charaf-Eddin, A. D'Aléo, G. Canard, D. Jacquemin, O. Siri, Extendable nickel complex tapes that reach NIR absorptions. Chem. Commun. 50, 15140 15143 (2014).

14. T. Isono, H. Kamo, A. Ueda, K. Takahashi, A. Nakao, R. Kumai, H. Nakao, K.

Kobayashi, Y. Murakami, H. Mori, Hydrogen bond-promoted metallic state in a purely organic single-component conductor under pressure. Nat. Commun. 4, 1344 (2013).

15. V. Augustyn, J. Come, M. A. Lowe, J. W. Kim, P. Taberna, S. H. Tolbert, H. D. Abruña, P. Simon, B. Dunn, High-rate electrochemical energy storage through $\mathrm{Li}^{+}$intercalation pseudocapacitance. Nat. Mater. 12, 518-522 (2013).

16. J. P. Zheng, P. J. Cygan, T. R. Jow, Hydrous ruthenium oxide as an electrode material for electrochemical capacitors. J. Electroanal. Chem. 142, 2699-2703 (1995).

17. L. Burke, O. J. Murphy, J. F. O'Neill, S. Venkatesan, The oxygen electrode Part 8Oxygen evolution at ruthenium dioxide anodes. J. Chem. Soc. Faraday Trans. 1. 73, 1659-1671 (1977).

18. M. R. Lukatskaya, O. Mashtalir, C. E. Ren, Y. D. Agnese, M. W. Barsoum, Y. Gogotsi, Cation intercalation and high volumetric capacitance of two-dimensional titanium carbide. Science 341, 1502-1505 (2013).

19. Y. Li, H. Shao, Z. Lin, J. Lu, L. Liu, B. Duployer, P. O. Å. Persson, P. Eklund, L. Hultman, M. Li, K. Chen, X. Zha, S. Du, P. Rozier, Z. Chai, E. Raymundo-piñero, P. Taberna, P. Simon, Q. Huang, A general Lewis acidic etching route for preparing MXenes with enhanced electrochemical performance in non-aqueous electrolyte. Nat. Mater. 19, 894-899 (2020).

20. X. Wang, T. S. Mathis, K. Li, Z. Lin, L. Vlcek, T. Torita, N. C. Osti, C. Hatter, P. Urbankowski, A. Sarycheva, M. Tyagi, E. Mamontov, P. Simon, Y. Gogotsi, Influences from solvents on charge storage in titanium carbide MXenes. Nat. Energy 4, 241-248 (2019).

21. H. Banda, J.-H. Dou, T. Chen, N. J. Libretto, M. Chaudhary, G. M. Bernard, J. T. Miller, V. K. Michaelis, M. Dincă, High-capacitance pseudocapacitors from $\mathrm{Li}^{+}$ion intercalation in nonporous, electrically conductive 2D coordination polymers. J. Am. Chem. Soc. 143, 2285-2292 (2021). 
22. T. S. Mathis, N. Kurra, X. Wang, D. Pinto, P. Simon, Energy storage data reporting in perspective - guidelines for interpreting the performance of electrochemical energy storage systems. Adv. Energy Mater. 9, 1902007 (2019).

23. C. Han, H. Li, Y. Li, J. Zhu, C. Zhi, Proton-assisted calcium-ion storage in aromatic organic molecular crystal with coplanar stacked structure. Nat. Commun. 12, 2400 (2021).

24. C. Wang, C. Jiang, Y. Xu, L. Liang, M. Zhou, J. Jiang, S. Singh, H. Zhao, A. Schober, Y. Lei, A selectively permeable membrane for enhancing cyclability of organic sodium-ion batteries. Adv. Mater. 28, 9182-9187 (2016).

25. H. Banda, J. Dou, T. Chen, Y. Zhang, M. Dincă, Dual-ion intercalation and high volumetric capacitance in a two- dimensional non-porous coordination polymer. Angew. Chem. Int. Ed. 60, 27119-27125 (2021).

26. G. S. Gautam, P. Canepa, W. D. Richards, R. Malik, G. Ceder, Role of structural $\mathrm{H}_{2} \mathrm{O}$ in intercalation electrodes: the case of $\mathrm{Mg}$ in nanocrystalline Xerogel- $\mathrm{V}_{2} \mathrm{O}_{5}$. Nano Lett. 16, 2426-2431 (2016).

27. X. Ji, J. Chen, F. Wang, W. Sun, Y. Ruan, L. Miao, J. Jiang, C. Wang, Water-activated $\mathrm{VOPO}_{4}$ for magnesium ion batteries. Nano Lett. 18, 6441-6448 (2018).

28. J. Shin, D. S. Choi, H. J. Lee, Y. Jung, J. W. Choi, Hydrated intercalation for highperformance aqueous zinc ion batteries. Adv. Energy Mater. 9, 1900083 (2019).

29. X. Wu, J. J. Hong, W. Shin, L. Ma, T. Liu, X. Bi, Y. Yuan, Y. Qi, T. W. Surta, W. Huang, J. Neuefeind, T. Wu, P. A. Greaney, J. Lu, X. Ji, Diffusion-free Grotthuss topochemistry for high-rate and long-life proton batteries. Nat. Energy 4, 123-130 (2019).

30. S. Wen, J. Lee, I. Yeo, J. Park, S. Mho, The role of cations of the electrolyte for the pseudocapacitive behavior of metal oxide electrodes, $\mathrm{MnO}_{2}$ and $\mathrm{RuO}_{2}$. Electrochim. Acta. 50, 849-855 (2004).

31. W. Li, S. Lee, A. Manthiram, High-nickel NMA: a cobalt-free alternative to NMC and NCA cathodes for lithium-ion batteries. Adv. Mater. 32, 2002718 (2020).

32. J. C. Russell, V. A. Posey, J. Gray, R. May, D. A. Reed, H. Zhang, L. E. Marbella, M. L. Steigerwald, Y. Yang, X. Roy, C. Nuckolls, S. R. Peurifoy, High-performance organic pseudocapacitors via molecular contortion. Nat. Mater. 20, 1136-1141 (2021). 
33. H. Ma, D. Kong, Y. Xu, X. Xie, Y. Tao, Z. Xiao, W. Lv, H. D. Jang, J. Huang, Q-H. Yang, Disassembly-reassembly approach to $\mathrm{RuO}_{2}$ /graphene composites for ultrahigh volumetric capacitance supercapacitor. Small 13, 1701026 (2017).

34. L. Suo, O. Borodin, T. Gao, M. Olguin, J. Ho, X. Fan, C. Luo, C. Wang, K. Xu, "Waterin-salt" electrolyte enables high-voltage aqueous lithium-ion chemistries. Science 350, 938-943 (2015).

35. D. Chao, S. Qiao, Toward high-voltage aqueous batteries: super- or low- concentrated electrolyte? Joule 4, 1846-1851 (2020).

36. P. Jaumaux, X. Yang, B. Zhang, J. Safaei, X. Tang, D. Zhou, C. Wang, G. Wang, Localized water-in-salt electrolyte for aqueous lithium-ion batteries. Angew. Chem. Int. Ed. 60, 19965-19973 (2021).

Acknowledgments: We thank Dr. Chenyue Sun for help with Raman measurements, Dr. Jianming Bai for help with PDF measurements, Dr. Yong Zhang for help with EDS measurements, and Dr. Ruperto Mariano and Dr. Justin Andrews for valuable discussions. Funding: This work was supported by Automobili Lamborghini S.p.A. Part of this work (SEM, XPS, and Raman) made use of the MRSEC Shared Experimental Facilities at MIT, supported by the National Science Foundation under award no. 1419807. The cRED data was collected at the Electron Microscopy Center (EMC), Department of Materials and Environmental Chemistry (MMK) in Stockholm University with the support of the Knut and Alice Wallenberg Foundation (KAW, 2012-0112) through the 3DEM-NATUR project. This research used resources of the Center for Functional Nanomaterials, the SMI beamline (12ID), and beamline 28-ID-2 (XPD) of the National Synchrotron Light Source II, supported by U.S. DOE Office of Science Facilities at Brookhaven National Laboratory under Contract No. DE-SC0012704. Cryo-EM specimens were prepared and imaged at the Automated Cryogenic Electron Microscopy Facility in MIT.nano on a Talos Arctica microscope, a gift from the Arnold and Mabel Beckman Foundation.

Author contributions: TC and MD conceived the idea. TC designed, synthesized and characterized BTABQ and pBTABQ. TC and HB performed and interpreted electrochemistry studies, in-situ and ex-situ characterizations. YL performed EPR studies. JL conducted the cRED measurements. YZ assisted the in-situ WAXS measurements. RP contributed to 
conception and development of the project. All authors interpreted the results and wrote the manuscript.

Competing interests: M.D., H.B., T.C. have filed a U.S. Patent (application number PCT/US2022/013925).

Data and materials availability: BTABQ and $\mathrm{pBTABQ}$ are available from Massachusetts Institute of Technology under a materials transfer agreement (MTA) with the university. All data are available in the main text or the supplementary materials. Crystallographic parameters for the structure of BTABQ is archived at the Cambridge Crystallographic Data Center (CCDC) (www.ccdc.cam.ac.uk) under reference nos. CCDC 2144223.

\section{Supplementary Materials}

Figs. S1 to S52

Tables S1 to S4 